\title{
A note on nontrivial intersection for selfmaps of complex Grassmann manifolds
}

\author{
Thaís F. M. Monis \\ tfmonis@rc.unesp.br
}

\author{
Northon C. L. Penteado \\ northoncanevari@gmail.com
}

Peter Wong*

pwong@bates.edu

\author{
Sérgio T. Ura \\ sergioura@gmail.com
}

\begin{abstract}
Let $G(k, n)$ be the complex Grassmann manifold of $k$-planes in $\mathbb{C}^{k+n}$. In this note, we show that for $1<k<n$ and for any selfmap $f: G(k, n) \rightarrow G(k, n)$, there exists a $k$-plane $V^{k} \in G(k, n)$ such that $f\left(V^{k}\right) \cap V^{k} \neq\{0\}$.
\end{abstract}

\section{Introduction}

The problem of determining the fixed point property (f.p.p.) for Grassmann manifolds has been studied by many authors (for example [7, [5], 6]).

Let

$$
\mathbb{F} M\left(n_{1}, \ldots, n_{k}\right)=\frac{U_{\mathbb{F}}(n)}{U_{\mathbb{F}}\left(n_{1}\right) \times \cdots \times U_{\mathbb{F}}\left(n_{k}\right)},
$$

$n_{1}+\cdots+n_{k}=n$. Here, $\mathbb{F}$ stands for one of the fields $\mathbb{R}, \mathbb{C}$ or the skew field $\mathbb{H}$, and

$$
U_{\mathbb{F}}(n)=\left\{\begin{array}{l}
O(n) \text { the orthogonal group of order } n \text { if } \mathbb{F}=\mathbb{R} \\
U(n) \text { the unitary group of order } n \text { if } \mathbb{F}=\mathbb{C}, \\
S p(n) \text { the symplectic group of order } n \text { if } \mathbb{F}=\mathbb{H} .
\end{array}\right.
$$

In [4], Glover and Homer have given the following necessary condition for $\mathbb{F} M\left(n_{1}, \ldots, n_{k}\right)$ to have the f.p.p..

Theorem 1 ([4], Theorem 1). If $\mathbb{F} M\left(n_{1}, \ldots, n_{k}\right)$ has the f.p.p., then $n_{1}, \ldots, n_{k}$ are distinct integers and, if $\mathbb{F}=\mathbb{R}$ or $\mathbb{C}$, at most one is odd.

The above theorem gives rise to the following conjectures:

${ }^{*}$ This work was supported by Capes of Brazil - Programa Pesquisador Visitante Especial - Ciência sem fronteiras, Grant number 88881.068085/2014-01. 
Conjecture 1. If $n_{1}, \ldots, n_{k}$ are all distinct then $\mathbb{H} M\left(n_{1}, \ldots, n_{k}\right)$ has the f.p.p..

Conjecture 2. If $n_{1}, \ldots, n_{k}$ are all distinct and at most one is odd then $\mathbb{F} M\left(n_{1}, \ldots, n_{k}\right)$ has the f.p.p., for $\mathbb{F}=\mathbb{R}$ and $\mathbb{F}=\mathbb{C}$.

The above conjectures were already proved to be true in the following cases:

- Projective spaces $(\mathbb{F} M(1, n-1))$;

- If $n_{2}$ and $n_{3}$ are distinct positive even integers and $n_{3} \geq 2 n_{2}^{2}-1$ then $\mathbb{C} M\left(1, n_{2}, n_{3}\right)$ has the f.p.p. ([4]).

- If $1, n_{2}$ and $n_{3}$ are distinct positive integers and $n_{3} \geq 2 n_{2}^{2}-1$, then $\mathbb{H} M\left(1, n_{2}, n_{3}\right)$ has the f.p.p. ([4]).

- If $n_{2}<n_{3}$ are even integers greater than 1 and either $n_{2} \leq 6$ or $n_{3} \geq n_{2}^{2}-2 n_{2}-2$, then $\mathbb{R} M\left(1, n_{2}, n_{3}\right)$ has the f.p.p. ([4]).

- If $n_{1}, n_{2}, n_{3}$ are positive integers such that at most one is odd, $n_{1} \leq 3, n_{3} \geq n_{2}^{2}-1$, and $\left[n_{1} / 2\right]<\left[n_{2} / 2\right]<\left[n_{3} / 2\right]$, then $\mathbb{R} M\left(n_{1}, n_{2}, n_{3}\right)$ has the f.p.p. ([4]).

- If $\mathbb{F}=\mathbb{C}$ or $\mathbb{H}, \mathbb{F} M(2, q)$ has the f.p.p. for all $q>2([7])$.

- $\mathbb{R}(2, q)$ has the f.p.p. for all $q=4 k$ or $q=4 k+1, k=1,2,3, \ldots$ ([7]).

- For $p \leq 3$ and $q>p$ or $p>3$ and $q \geq 2 p^{2}-p-1, \mathbb{C} M(p, q)$ has the f.p.p. iff $p q$ is even ([5]).

- For $p \leq 3$ and $q>p$ or $p>3$ and $q \geq 2 p^{2}-p-1, \mathbb{H} M(p, q)$ always has the f.p.p. ([5]).

The main tool used to prove the above results is the calculation of the Lefschetz number of a self-map of such a space. Let's focus on the case of complex Grassmann manifolds $\mathbb{C}(k, n)=$ $G(k, n)$, the space of $k$-planes in $\mathbb{C}^{k+n}$. Let $\gamma^{k}$ be the canonical $k$-plane bundle over $G(k, n)$. If

$$
\operatorname{ch}\left(\gamma^{k}\right)=1+c_{1}+\cdots+c_{k}, \quad c_{i} \in H^{2 i}(G(k, n) ; \mathbb{Q}),
$$

is the total Chern class of $\gamma^{k}$, then the cohomology ring $H^{*}(G(k, n) ; \mathbb{Q})$ is given by:

$$
H^{*}(G(k, n) ; \mathbb{Q})=\mathbb{Q}\left[c_{1}, \ldots, c_{k}\right] / I_{k, n}
$$


where $I_{k, n}$ is the ideal generated by the elements $\left(c^{-1}\right)_{n+1}, \ldots,\left(c^{-1}\right)_{n+k}$. Here, $\left(c^{-1}\right)_{q}$ is the part of the formal inverse of $c$ in dimension $2 q$ (see [6], Theorem 2.1). Then, $c_{1}$ is the only generator in dimension 2. Therefore, given a self-map $f: G(k, n) \rightarrow G(k, n), f^{*}\left(c_{1}\right)=m c_{1}$ for some coefficient $m$.

Theorem 2 ([5], Theorem 1). Let $k \leq 3$ and $n>k$ or $k>3$ and $n \geq 2 k^{2}-k-1$. Then every graded ring endomorphism of $H^{*}(G(k, n) ; \mathbb{Q})$ is an Adams endomorphism 1 . Consequently, if $f: G(k, n) \rightarrow G(k, n)$ is a self-map with $f^{*}\left(c_{1}\right)=m c_{1}$ then $f^{*}\left(c_{i}\right)=m^{i} c_{i}, i=1, \ldots, k$.

The classification of the graded ring endomorphisms of $H^{*}(G(k, n) ; \mathbb{Q})$ is fundamental in the study of f.p.p. for $G(k, n)$ because of the following.

Proposition 1. An Adams endomorphism of $H^{*}(G(k, n) ; \mathbb{Q})$ has Lefschetz number zero if and only if its degree is -1 and $k n$ is odd.

Proof. See [4, Proposition 4.

In [6], M. Hoffman was able to prove the following.

Theorem 3 ([6], Theorem 1.1). Let $k<n$ and $h$ be a graded ring endomorphism of $H^{*}(G(k, n) ; \mathbb{Q})$ with $h\left(c_{1}\right)=m c_{1}, m \neq 0$. Then $h\left(c_{i}\right)=m^{i} c_{i}, 1 \leq i \leq k$.

If $k<n$ and $h$ is a graded ring endomorphism of $H^{*}(G(k, n) ; \mathbb{Q})$ with $h\left(c_{1}\right)=0$, it is still unclear about what $h$ looks like in general. The conjecture is that, in this case, $h$ must be the null homomorphism. If one can prove such conjecture then the problem of determining the f.p.p. for $G(k, n)$ will be completely solved.

In this note, we prove a much more modest result for complex Grassmann manifolds than a fixed point theorem. Our main theorem is the following.

Theorem 4 (Main Result). Let $k>1$ and $k<n$. Then for every continuous map $f: G(k, n) \rightarrow$ $G(k, n)$ there exists a $k$-plane $V^{k} \in G(k, n)$ such that $V^{k} \cap f\left(V^{k}\right) \neq\{0\}$.

\footnotetext{
${ }^{1}$ An Adams endomorphism of $H^{*}(G(k, n) ; \mathbb{Q})$ is a endomorphism $\varphi$ of the form $\varphi(x)=\lambda^{i} x$ for $x \in$ $H^{2 i}(G(k, n) ; \mathbb{Q})$. The coefficient $\lambda$ is called the degree of $\varphi$.
} 
The motivation for this work is the paper 8 , where the author gave an alternative proof for the f.p.p. of $\mathbb{C} P^{2 n}$ using characteristic classes. In fact, a closer look at the proof of the main result in $[8]$ indicates that the same argument would also yield an alternative proof of the f.p.p. for $\mathbb{R} P^{2 n}$ by replacing Chern classes with Stiefel-Whitney classes. We should also point out that a non-trivial intersection result similar to Theorem 4 has been obtained in [1] for maps between two different Grassmann manifolds.

\section{Proof of the Main Theorem}

Throughout this paper, $G(k, n)$ denotes the complex Grassmann manifold of $k$-planes in $\mathbb{C}^{k+n}$.

Note that, since $G(k, n)$ and $G(n, k)$ are homeomorphic, $\gamma^{k}$ and $\gamma^{n}$ can be seen as subbundles of the trivial bundle $G(k, n) \times \mathbb{C}^{k+n}$, which is denoted by $\epsilon^{k+n}$, and, under such identification,

$$
\gamma^{k} \oplus \gamma^{n}=\epsilon^{k+n}
$$

Lemma 1. Let $\operatorname{ch}\left(\gamma^{n}\right)=1+\bar{c}_{1}+\cdots+\bar{c}_{n}$ be the total Chern class of the bundle $\gamma^{n}$. Then, a general formula for the class $\bar{c}_{i}$ in terms of the Chern classes of $\gamma^{k}$ is given by

$$
\bar{c}_{i}=\sum_{\|\alpha\|=i}(-1)^{|\alpha|} \frac{|\alpha| !}{\alpha !} \operatorname{ch}\left(\gamma^{k}\right)^{\alpha},
$$

where $\alpha$ represents the $k$-uple $\alpha=\left(a_{1}, \ldots, a_{k}\right),\|\alpha\|=a_{1}+2 a_{2}+\cdots+k a_{k},|\alpha|=a_{1}+a_{2}+\cdots+a_{k}$, $\alpha !=a_{1} ! a_{2} ! \cdots a_{k} !$ and $\operatorname{ch}\left(\gamma^{k}\right)^{\alpha}=c_{1}^{a_{1}} \smile c_{2}^{a_{2}} \smile \cdots \smile c_{k}^{a_{k}}$.

Proof. The proof is given recursively in the index $i$.

As $\gamma^{k} \oplus \gamma^{n}=\epsilon^{k+n}$, we have

$$
\operatorname{ch}\left(\gamma^{k}\right) \smile \operatorname{ch}\left(\gamma^{n}\right)=\operatorname{ch}\left(\epsilon^{k+n}\right)=1
$$

in $H^{*}(G(k, n) ; \mathbb{Z})$. So

$$
\left(1+c_{1}+\cdots+c_{k}\right) \smile\left(1+\bar{c}_{1}+\cdots+\bar{c}_{n}\right)=1
$$

and then

$$
\begin{aligned}
& 1=1 \\
& 0=c_{1}+\bar{c}_{1} \\
& 0=c_{2}+c_{1} \smile \bar{c}_{1}+\bar{c}_{2}
\end{aligned}
$$


Then

$$
\bar{c}_{j}=-\sum_{i=1}^{j} c_{i} \smile \bar{c}_{j-i}
$$

for all $j=1, \ldots, n$, with the convention $c_{i}=0$ when $i>k$. Thus,

(i) $\bar{c}_{1}=-c_{1}$;

(ii) $\bar{c}_{2}=-\left(c_{1} \smile-c_{1}\right)-c_{2}=c_{1}^{2}-c_{2}$;

(iii) Suppose

$$
\bar{c}_{j}=\sum_{\|\alpha\|=j}(-1)^{|\alpha|} \frac{|\alpha| !}{\alpha !} \operatorname{ch}\left(\gamma^{k}\right)^{\alpha},
$$

for $j=1, \ldots, m-1<n$.

Then

$$
\begin{aligned}
\bar{c}_{m} & =-\sum_{i=1}^{m} c_{i} \smile \bar{c}_{m-i} \\
& =-\sum_{i=1}^{m}\left(c_{i} \smile \sum_{\|\alpha\|=m-i}(-1)^{|\alpha|} \frac{|\alpha| !}{\alpha !} \operatorname{ch}\left(\gamma^{k}\right)^{\alpha}\right) \\
& =\sum_{i=1}^{m}\left(c_{i} \smile \sum_{\|\alpha\|=m-i}(-1)^{|\alpha|+1} \frac{|\alpha| !}{\alpha !} \operatorname{ch}\left(\gamma^{k}\right)^{\alpha}\right) \\
& =\sum_{i=1}^{m}\left(\sum_{\|\alpha\|=m-i}(-1)^{|\alpha|+1} \frac{|\alpha| !}{\alpha !} \operatorname{ch}\left(\gamma^{k}\right)^{\alpha} \smile c_{i}\right) \\
& =\sum_{i=1}^{m} \sum_{\|\alpha\|=m-i}(-1)^{\left|\alpha+e_{i}\right|} \frac{|\alpha| !}{\alpha !} \operatorname{ch}\left(\gamma^{k}\right)^{\alpha+e_{i}} \quad\left(e_{i}=(0, \ldots, 0,1,0, \ldots 0)\right) \\
& =\sum_{\|\beta\|=m}(-1)^{|\beta|} X(\beta) \operatorname{ch}\left(\gamma^{k}\right)^{\beta} \quad\left(\beta=\alpha+e_{i}\right)
\end{aligned}
$$


where

$$
\begin{aligned}
X(\beta) & =\sum_{b_{i} \neq 0} \frac{\left|\beta-e_{i}\right| !}{\left(\beta-e_{i}\right) !} \\
& =\sum_{b_{i} \neq 0} \frac{(|\beta|-1) ! b_{i}}{\beta !} \\
& =\sum_{i=1}^{m} \frac{(|\beta|-1) ! b_{i}}{\beta !} \\
& =\frac{(|\beta|-1) ! \sum_{i=1}^{m} b_{i}}{\beta !} \\
& =\frac{(|\beta|-1) !|\beta|}{\beta !} \\
& =\frac{|\beta| !}{\beta !}
\end{aligned}
$$

\subsection{Proof of Theorem 4}

Suppose, to the contrary, there exists a continuous map $f: G(k, n) \rightarrow G(k, n)$ such that $V^{k} \cap$ $f\left(V^{k}\right)=\{0\}$ for every $k$-plane $V^{k} \in G(k, n)$. Then the direct sum $\gamma^{k} \oplus f^{*} \gamma^{k}$ can be seen as a subbundle of the trivial bundle $\epsilon^{k+n}$. Let $\eta^{n-k}$ be the normal bundle of $\gamma^{k} \oplus f^{*} \gamma^{k}$ in $\epsilon^{k+n}$. Then

$$
\operatorname{ch}\left(\gamma^{k}\right) \smile \operatorname{ch}\left(f^{*} \gamma^{k}\right) \smile \operatorname{ch}\left(\eta^{n-k}\right)=1 .
$$

It follows that

$$
\operatorname{ch}\left(f^{*} \gamma^{k}\right) \smile \operatorname{ch}\left(\eta^{n-k}\right)=1+\bar{c}_{1}+\cdots+\bar{c}_{n} .
$$

Let

$$
\operatorname{ch}\left(f^{*} \gamma^{k}\right)=1+\tilde{c}_{1}+\cdots+\tilde{c}_{k}, \tilde{c}_{i} \in H^{2 i}(G(k, n) ; \mathbb{Q}),
$$

and

$$
\operatorname{ch}\left(\eta^{n-k}\right)=1+t_{1}+\cdots+t_{n-k}, t_{j} \in H^{2 j}(G(k, n) ; \mathbb{Q}) .
$$

We will show that it is impossible for

$$
\bar{c}_{n}=\tilde{c}_{k} \smile t_{n-k} .
$$

The proof of the impossibility of the above equality will be split into several cases. 
Case 1: $1<k \leq 3$. Since $c_{1} \in H^{2}(G(k, n) ; \mathbb{Q})$ is the only generator in dimension $2, f^{*}\left(c_{1}\right)$ is a multiple of $c_{1}$, let's say $f^{*}\left(c_{1}\right)=m c_{1}$. Following [7] and [5], for $k \leq 3$ and $k<n$, every endomorphism of the ring $H^{*}(G(k, n) ; \mathbb{Q})$ that preserves dimension is an Adams endomorphism. Therefore, if $f^{*}\left(c_{1}\right)=m c_{1}$ then $f^{*}\left(c_{2}\right)=m^{2} c_{2}, \ldots, f^{*}\left(c_{k}\right)=m^{k} c_{k}$. Thus

$$
\operatorname{ch}\left(f^{*} \gamma^{k}\right)=f^{*}\left(\operatorname{ch}\left(\gamma^{k}\right)\right)=1+m c_{1}+m^{2} c_{2}+\cdots+m^{k} c_{k} .
$$

It follows that

$$
\bar{c}_{n}=m^{k} c_{k} \smile t_{n-k},
$$

in contradiction with Lemma 1,

Case 2: $k>3$. This case will be split in four cases.

Case 2(i): $n=l(k-1)+r$ with remainder $r \neq 1$, that is, $1<r<k-1$ or $r=0$. In this case, $r$ is of the form $r=2 i$ or $r=2 i+3$, for some integer $i \geq 0$. In case of $r=2 i$, the class $c_{k-1}^{l} \smile c_{2}^{i}$ does not appear in $\tilde{c}_{k} \smile t_{n-k}$ but, by Lemma 1, it appears in $\bar{c}_{n}$, contradicting $\bar{c}_{n}=\tilde{c}_{k} \smile t_{n-k}$. In case of $r=2 i+3$, the class $c_{k-1}^{l} c_{2}^{i} c_{3}$ does not appear in $\tilde{c}_{k} \smile t_{n-k}$ but, by Lemma 1, it appears in $\bar{c}_{n}$, contradicting $\bar{c}_{n}=\tilde{c}_{k} \smile t_{n-k}$.

Case 2(ii): $k>4$ and $n=(l+1)(k-1)+1$. In this case, we have

$$
\begin{aligned}
n & =(l+1)(k-1)+1 \\
& =l(k-1)+k
\end{aligned}
$$

and, since $n>k, l \geq 1$. We can write $n=(l+1)(k-1)+1$ in the form

$$
n=(l-1)(k-1)+2(k-2)+3
$$

and, since we are supposing $k>4, k-2>2$. With these information, one can check that the class $c_{k-1}^{m-1} \smile c_{k-2}^{2} \smile c_{3}$ cannot appear in $\tilde{c}_{k} \smile t_{n-k}$. On the other hand, by Lemma1, the class $c_{k-1}^{m-1} \smile c_{k-2}^{2} \smile c_{3}$ appears in $\bar{c}_{n}$. Therefore, $\bar{c}_{n}=\tilde{c}_{k} \smile t_{n-k}$ is impossible.

Case 2(iii): $k=4, n=(l+1)(k-1)+1$ and $l$ even, say $l=2 j$. In this case, $n-k=3 l$ and, since $n>1, l \geq 1$. Let

$$
\begin{aligned}
\tilde{c}_{4} & =c_{1}^{4}+\alpha c_{2}^{2}+\theta c_{4}+\text { other terms } \\
t_{3 l} & =c_{1}^{3 l}+\alpha^{\prime} c_{2}^{3 j}+\beta c_{3}^{l}+\text { other terms. }
\end{aligned}
$$


Thus, in the product $\tilde{c}_{4} \smile t_{3 l}, \alpha \alpha^{\prime}$ is the coefficient of $c_{2}^{3 j+2}, \alpha \beta$ is the coefficient of $c_{2}^{2} \smile c_{3}^{l}$ and $\theta \beta$ is the coefficient of $c_{4} \smile c_{3}^{l}$. From Lemma 1 together with the fact that $\tilde{c}_{4} \smile t_{3 l}=\bar{c}_{n}$, it follows that

$$
\begin{aligned}
\alpha \alpha^{\prime} & =\frac{(3 j+2) !}{(3 j+2) ! 1 !} \\
\alpha \beta & =\frac{(l+2) !}{l ! 2 !} \\
\theta \beta & =\frac{(l+1) !}{l ! 1 !} .
\end{aligned}
$$

Thus

$$
\begin{aligned}
\alpha \alpha^{\prime} & =1 \\
\alpha \beta & =\frac{(l+2)(l+1)}{2} \\
\theta \beta & =l+1 .
\end{aligned}
$$

Then, we conclude that $\alpha= \pm 1, \beta= \pm \frac{(l+2)(l+1)}{2}$ and $|\beta|=\frac{(l+2)(l+1)}{2}$ divides $\theta \beta=l+1$. It follows that $l=0$, but $l \geq 1$, a contradiction!

Case 2(iv): $k=4, n=(l+1)(k-1)+1$ and $l$ odd, say $l=2 j+1$. Again, $n-k=3 l$ and, since $n>1, l \geq 1$. Let

$$
\begin{aligned}
\tilde{c}_{4} & =c_{1}^{4}+\alpha c_{2}^{2}+\theta c_{4}+\gamma c_{1} c_{3}+\text { other terms } \\
t_{3 l} & =c_{1}^{3 l}+\alpha^{\prime} c_{1} c_{2}^{3 j+1}+\beta c_{3}^{l}+\text { other terms. }
\end{aligned}
$$

It follows that, in the product $\tilde{c}_{4} \smile t_{3 l}, \alpha \alpha^{\prime}$ is the coefficient of $c_{1} \smile c_{2}^{3 j+3}, \alpha \beta$ is the coefficient of $c_{2}^{2} \smile c_{3}^{l}, \theta \beta$ is the coefficient of $c_{4} \smile c_{3}^{l}$ and $\gamma \beta$ is the coefficient of $c_{1} \smile c_{3}^{l+1}$. Since $\bar{c}_{n}=\tilde{c}_{4} \smile t_{3 l}$, together with Lemma 1,

$$
\begin{aligned}
\alpha \alpha^{\prime} & =\frac{(3 j+4) !}{1 !(3 j+3) !} \\
\alpha \beta & =\frac{(l+2) !}{l ! 2 !} \\
\theta \beta & =\frac{(l+1) !}{l ! 1 !} \\
\gamma \beta & =\frac{(l+2) !}{1 !(l+1) !} .
\end{aligned}
$$

Thus 


$$
\begin{aligned}
\alpha \alpha^{\prime} & =3 j+4 \\
\alpha \beta & =\frac{(l+2)(l+1)}{2} \\
\theta \beta & =l+1 \\
\gamma \beta & =l+2 .
\end{aligned}
$$

From the two last equalities above, it follows that $\beta$ divides $l+1$ and $l+2$. Therefore, $\beta=1$. It follows that $\alpha=\frac{(l+2)(l+1)}{2}$ and, since $\alpha$ divides $3 j+4$,

$$
\frac{(l+2)(l+1)}{2} \leq 3 j+4=\frac{3 l+5}{2} .
$$

Therefore, $l^{2} \leq 3$. Since $l$ is an integer not smaller than 1 , it follows that $l=1$. Then, $3 j+4=\frac{3 l+5}{2}=4$ is divisible by $\frac{(l+2)(l+1)}{2}=3$, a contradiction!

\section{References}

[1] Chakraborty, Prateep and Sankaran, Parameswaran Maps between certain complex Grassmann manifolds. Topology Appl. 170 (2014), 119-123.

[2] Duan, Haibao, Self-maps of the Grassmannian of complex structures. Compositio Math. 132 (2002), no. 2, 159-175.

[3] Glover, Henry and Homer, William, Self-maps of flag manifolds. Trans. Amer. Math. Soc. 267 (1981), no. 2, 423-434.

[4] Glover, Henry and Homer, William, Fixed points on flag manifolds, Pacific J. Math. 101 (1982), no. 2, 303-306.

[5] Glover, Henry and Homer, William, Endomorphisms of the cohomology ring of finite Grassmann manifolds. Lecture Notes in Math., vol. 657, Springer-Verlag, Berlin and New York, 1978, 179-193.

[6] Hoffman, Michael, Endomorphisms of the cohomology of complex Grassmannians. Trans. Amer. Math. Soc. 281 (1984), 745-740.

[7] O’Neill, Larkin S., On the f.p.p. for Grassmann manifolds. Ph.D. Thesis, Ohio State University, 1974.

[8] Taghavi, Ali, An alternative proof for the f.p.p. of $\mathbb{C} P^{2 n}$. Expo. Math. 33 (2015), 105-107. 


\section{Thaís F. M. Monis}

Departamento de Matemática, IGCE, Univ Estadual Paulista. email: tfmonis@rc.unesp.br

\section{Northon C. L. Penteado}

Departamento de Matemática, IGCE, Univ Estadual Paulista. email: northoncanevari@gmail.com

\section{Sérgio T. Ura}

Departamento de Matemática, IGCE, Univ Estadual Paulista.

email: sergioura@gmail.com

\section{Peter Wong}

Department of Mathematics, Bates College

e-mail: pwong@bates.edu 